arXiv: 0711.4637v6[nucl-th]

\title{
A nonperturbative parametrization and scenario for EFT renormalization
}

\author{
Ji-Feng Yang \\ Department of Physics, East China Normal University, Shanghai, 200062, China
}

(Dated: March 10, 2009)

\begin{abstract}
We present a universal form of the $T$-matrices renormalized in nonperturbative regime and the ensuing notions and properties that fail conventional wisdoms. A universal scale is identified and shown to be renormalization group invariant. The effective range parameters are derived in a nonperturbative scenario with some new predictions within the realm of contact potentials. Some controversies are shown to be due to the failure of conventional wisdoms.
\end{abstract}

Applications of the effective field theory (EFT) methods now prevail in physical literature. In particular, the applications of the EFT approach to nucleon systems has been producing many encouraging results [1], pointing towards a promising field theoretical framework for nuclear system. However, the nonperturbative nature makes the renormalization of such EFT's rather nontrivial and still creates controvsersies [2, 3, 4] to be settled. Sufficient evidences have been accumulated that the conventional wisdoms for renormalization cease to apply straightforwardly in nonperturbative regimes. This is not unexpected as they are established within perturbative frameworks. Therefore it is desirable to reveal novel notions and aspects of renormalization that deviate from the conventional wisdoms. In particular, it is desirable to obtain more concrete parametrization of the prescription dependence of the objects (here, the $T$-matrix) in nonperturbative regimes as much as possible.

To this end, we work out the rigorous solutions of the $T$-matrices for low-energy nucleon-nucleon $(N N)$ scattering that solve the Lippmann-Schwinger equation (LSE) in all partial wave channels, using contact potentials constructed according to the chiral EFT approach [5]. The ${ }^{1} S_{0}$ channel has been worked out in Ref. [6] up to chiral order $\Delta=4$. Here, we present the universal forms for both uncoupled and coupled channels. Then it is immediate to see some features and notions that are intrinsically nonperturbative and deviate from the conventional wisdoms of renormalization within perturbative frameworks. These are important conceptual gainings that could resolve most of the controversies about the applications of EFT in nonperturbative regimes. The notions and scenario demonstrated below are naturally illuminating for any problem that is beset with nonperturbative divergences, especially in the systems governed by singular short-distance interactions. In addition, the analytical results and scenario presented here could also be seen as a field-theoretical solution to the universality of large scattering lengths in atomic and molecular systems [7].

According to Weinberg[5], the EFT approach to $N N$ scattering consists of two steps: First, the potentials for $N N$ scattering are systematically constructed using chiral perturbation theory $(\chi \mathrm{PT})$ as the relevant EFT up to certain chiral order $\Delta: \mathcal{O}\left(\left\{p, m_{\pi}\right\}^{\Delta} / \Lambda^{\Delta}\right)$ with $\Lambda$ being the upper limit for low energy $N N$ scattering (e.g., $\Lambda \sim 0.5 \mathrm{GeV}$ ); Second, the nonperturbative $N N$ scattering $T$-matrices could be obtained by solving the LSE with the potential constructed in the previous step. As LSE is hard to solve rigorously for pion-exchange potentials, we first work with contact potentials $(\operatorname{EFT}(\not))$ that facilitate rigorous solutions where the LSE for $L$-wave reduces to an algebraic form by employing the following factorization trick or ansatz [8]:

$$
\begin{aligned}
& V \equiv q^{L}\left(q^{\prime}\right)^{L} \sum_{i, j=0}^{\Delta / 2-L} \lambda_{i j} q^{2 i}\left(q^{\prime}\right)^{2 j}=q^{L}\left(q^{\prime}\right)^{L} U^{T}(q) \lambda U\left(q^{\prime}\right), \\
& T \equiv q^{L}\left(q^{\prime}\right)^{L} \sum_{i, j=0}^{\Delta / 2-L} \tau_{i j} q^{2 i}\left(q^{\prime}\right)^{2 j}=q^{L}\left(q^{\prime}\right)^{L} U^{T}(q) \tau U\left(q^{\prime}\right),
\end{aligned}
$$

with $U(q) \equiv\left(1, q^{2}, q^{4}, \cdots, q^{\Delta-2 L}\right)^{T}$ being a column vector and $q, q^{\prime}$ being the external off-shell momenta. This is because for pionless interactions, the $N N$ potential up to order $\Delta$ degrades into contact interactions that become a polynomial in terms of external momenta up to power $\Delta$. Here $\lambda$ denotes the real symmetric matrix comprises of the contact couplings $([C \ldots])$ as $V$ is symmetric with respect to the external momenta $q$ and $q^{\prime}$. For example, in ${ }^{1} S_{0}$ channels at $\Delta=2$, we have,

$$
\lambda=\left(\begin{array}{cc}
C_{0} & C_{2} \\
C_{2} & 0
\end{array}\right) .
$$


Then the algebraic LSE takes the following form:

$$
\tau(E)=\lambda+\lambda \mathcal{I}(E) \tau(E)
$$

where the matrix $\mathcal{I}(E)$ comprises of the integrals arising from convolution. Note that $\tau$ and $\mathcal{I}$ are symmetric matrices as $\lambda$ is. As $\mathcal{I}$ is complex, so is $\tau$. A general element of $\mathcal{I}$ can be parametrized as follows

$$
\begin{aligned}
\mathcal{I}_{i, j}(E) & \equiv\left\{\int \frac{d^{3} k}{(2 \pi)^{3}} \frac{k^{2(L+i+j-2)}}{E-k^{2} / M+i \epsilon}\right\}_{R}=\sum_{m=1}^{L+i+j-2} J_{2 m+1} p^{2(L+i+j-m-2)}-\mathcal{I}_{0} p^{2(L+i+j-2)}, \\
\mathcal{I}_{0} & \equiv J_{0}+i \frac{M}{4 \pi} p
\end{aligned}
$$

where the subscript $R$ denotes any possible regularization and/or renormalization prescription rendering the integrals finite and $\left[J_{0}, J_{2 m+1}\right]$ being the corresponding parametrization. This algebraic LSE is easy to solve. Then, for any uncoupled partial wave channel, the on-shell $T$-matrices could be readily obtained from $T=p^{2 L} U^{T}(p) \tau U(p)$ with $p=\sqrt{M E}[\underline{6}, 9]$, which can be simplified to the following form:

$$
\frac{1}{T_{L}}=\mathcal{I}_{0}+\frac{N_{L}\left([C \ldots],\left[J_{2 m+1}\right], p^{2}\right)}{D_{L}\left([C \ldots],\left[J_{2 m+1}\right], p^{2}\right) p^{2 L}}
$$

where $N_{L}$ and $D_{L}$ are polynomials in terms of the following real parameters: the couplings $[C \ldots]$, the constants $\left[J_{2 m+1}, m>0\right]$ and $p^{2}$. While for coupled channels, the inverse of the on-shell $T$-matrices would take the following form:

$$
\mathbf{T}_{J}^{-1}=\mathcal{I}_{0} \mathbf{I}+\boldsymbol{\Delta}_{J}, \quad \mathbf{I} \equiv\left(\begin{array}{ll}
1 & 0 \\
0 & 1
\end{array}\right), \quad \boldsymbol{\Delta}_{J} \equiv\left(\begin{array}{cc}
\frac{N_{J-1, J-1}}{D_{J-1, J-1} p^{2(J-1)}}, & \frac{-N_{J-1, J+1}}{D_{J-1, J+1} p^{2 J}} \\
\frac{-N_{J-1, J 1}}{D_{J-1, J+1} p^{2 J}}, & \frac{N_{J+1, J+1}}{D_{J+1, J+1} p^{2(J+1)}}
\end{array}\right) .
$$

Again $[N \ldots, D \ldots]$ are real polynomials in terms of $\left[C_{\ldots}.\right],\left[J_{2 m+1}, m>0\right]$ and $p$, hence (chiral) perturbative in nature. Note that unitarity is automatically satisfied here. At this stage, both the real part of $\mathcal{I}_{0}$ and the constants $\left[J_{2 m+1}, m>0\right]$ are prescription dependent. The overall factors of $p^{2 \cdots}$ have been factored out so that the expansion of $[N \ldots, D \ldots]$ in terms of $p^{2}$ starts from $p^{0}$. For example, the results for ${ }^{3} S_{1}-{ }^{3} D_{1}$ at chiral order $\Delta=4$ read,

$$
\mathbf{T}_{3 S_{1}-{ }^{3} D_{1}}^{-1}=\mathcal{I}_{0} \mathbf{I}+\Delta^{{ }^{3} S_{1}-{ }^{3} D_{1}}, \quad \Delta_{{ }^{3} S_{1}-{ }^{3} D_{1}} \equiv \frac{1}{\mathcal{D}_{1} p^{4}}\left(\begin{array}{cc}
\mathcal{N}_{1} p^{4}, & -\mathcal{D}_{s d} p^{2} \\
-\mathcal{D}_{s d} p^{2}, & \mathcal{D}_{0}
\end{array}\right)
$$

where $D_{0,2}=D_{2,2}=D_{0,0}$ and for convenience we introduced the following notations: $\mathcal{N}_{1} \equiv N_{0,0}, \quad \mathcal{D}_{1} \equiv$ $D_{0,0}, \quad \mathcal{D}_{s d} \equiv N_{0,2}, \quad \mathcal{D}_{0} \equiv N_{2,2}$. For details we refer to a forthcoming report [9].

Eqs.(5) and (6) exhibit the following important features: (1) First, the same complex parameter $\mathcal{I}_{0}$ appears in all channels in the same isolated position in $1 / T$ or $\mathbf{T}^{-1}$ and the rest parts of $1 / T$ or $\mathbf{T}^{-1}$ are independent of $\mathcal{I}_{0}$, i.e., $\mathcal{I}_{0}$ is "decoupled" from $[C \ldots]$ and $\left[J_{2 m+1}, m>0\right]$ in every channel*. This structure is most pivotal. (2) Second, with the potentials truncated at finite order, only finite many of $[J \ldots .$. (or finite types of divergences) enter the game, in spite that there are formally infinite many divergent items in the iteration of LSE. (3) Both $[N \ldots]$ and $[D \ldots]$ are chiral perturbative (or perturbative in the corresponding EFT).

Since the $p(=\sqrt{M E}$ )-dependence of the on-shell $T$-matrices (and hence the inverse of $T$-matrices) is physical, the prescription variations (i.e., variations of $[J \ldots]$ ) must be compensated by that of the couplings. This is nothing else but the general principle of renormalization group (RG) invariance, then appropriate combinations of the coefficients of $p$ in $[N \ldots]$ and $[D \ldots]$ must be RG invariants. The most outstanding point is that, the isolation or "decoupling" of $\mathcal{I}_{0}$ from $[C \ldots]$ and $\left[J_{2 m+1}, m>0\right]$ makes itself a renormalization group invariant parameter in all channels, hence, $J_{0}$ is a physical scale [6]. Therefore, $\mathcal{I}_{0}$ is in fact a fundamental and universal parameter in the low energy $N N$ scattering, and $J_{0}$ is no longer an ordinary renormalization

\footnotetext{
* The rigorous proof of this point for ${ }^{1} S_{0}$ channel has been given in Ref.[]. , which could be generalized to higher channels, we will give the detailed proof in a forthcoming report.
} 
scale. Such a RG invariant quantity was also predicted in Wilsonian RG approach [10], $\hat{V}_{0}$, whose inverse is just $-\operatorname{Re}\left(\mathcal{I}_{0}\right)=-J_{0}$ computed in the Wilsonian cutoff approach. This is not the only deviation from the conventional wisdoms for renormalization, according to which a divergent integral usually produces sliding scales that are physically meaningless within perturbative formulation.

In perturbative framework, the couplings in the contact potential would get renormalized and "run". At lowest order, similar notion is feasible in ${ }^{1} S_{0}$ channel[11]: $1 / T=J_{0}+i M p /(4 \pi)+1 / C_{0}=i M p /(4 \pi)+$ $M \mu /(4 \pi)+1 / C_{0 ; R}(\mu)$, where $J_{0}+1 / C_{0}=M \mu /(4 \pi)+1 / C_{0 ; R}(\mu)$ is RG invariant as $N_{0}$ is a constant here, actually $N_{0}=1$. But at higher orders (e.g., $\Delta \geq 4$ ), it is easy to see that the rational dependence of $T$ matrices upon $p$ precludes the conventional wisdoms from being feasible, i.e., it is no longer possible to let the variations in the prescription parameters $[J \ldots]$ be readily absorbed into the couplings and let the couplings "run". This point could be seen from the requirement that the appropriate combinations of the coefficients of $p$ in $[N \ldots, D \ldots]$ should be RG invariant, which in turn imposes strong constraints upon the variations in the couplings $[C \ldots]$ and $\left[J_{2 m+1}, m>0\right]$. ( $J_{0}$ is already excluded from the set of prescription parameters as it is an RG invariant scale now.) In fact, for ${ }^{1} S_{0}$ at $\Delta=4$, the coefficients of the highest power term in $N_{0}, D_{0}$ are respectively $N_{0 ; 2}=C_{4}^{2} J_{3}^{2}, D_{0,3}=-C_{4}^{2} J_{3}$, which could not make the ratios $N_{0 ; 2} / N_{0 ; 0}$ and $D_{0 ; 3} / N_{0 ; 0} \mathrm{RG}$ invariant at the same time, see Ref.[6] .

The second feature noted above engenders a novel notion of "finiteness": Only a finite number of nonperturbative divergences are to be removed in a manner preserving the functional dependence upon $[C \ldots]$ and $p$, which underlies the feasibility of renormalization with a few nonperturbative counterterms in Refs. 12, 13]. This 'finiteness' is a measure of nonperturbative renormalizability and not directly linked to EFT power counting. Hence nonperturbative counterterms (termed as "endogenous" in Ref. [6]) are not proportionate to perturbative ones that obey EFT power counting. This is in sheer contrast with perturbative renormalization programs, where consistency requires that the counterterms be introduced or constructed at exactly the same perturbation order as the divergent vertex in consideration. This is another place where the conventional wisdoms fail, usually interpreted as the "inconsistency" of Weinberg's power counting. We will return to this point later. This "finiteness" also underlies the feasibility of the finite cutoff approaches [14, 15, 16, 17, 18].

In fact the nonperturbative form of $T$-matrices and their RG invariance lead to an entanglement between the couplings and the prescription: They must be defined coherently in order to match physical boundaries. Then, the prescription must be appropriately defined after the couplings are given first. Below, to obtain unnaturally large scattering lengths and naturally sized effective range, etc., we suggest a simple and natural strategy: The original $\operatorname{EFT}(\chi \mathrm{PT})$ power counting for potential construction are kept intact, i.e., no modification of the power counting rules of the couplings $\left[C_{\Delta}\right]$; In the meantime, $J_{0}$ and $\left[J_{2 m+1}, m>0\right]$ are so determined that physical boundary conditions are fulfilled. Then, to yield large (unnatural) $S$-wave scattering lengths, the most 'natural' or simplest scenario would be as follows [6]:

$$
C_{\Delta} \sim 4 \pi /\left(M \Lambda^{\Delta+1}\right) ; \quad J_{0} \sim M \Lambda /(4 \pi) \sim\left|1 / C_{0}\right| ; \quad J_{2 m+1} \sim M \mu^{2 m+1} /(4 \pi), m>0,
$$

with $C_{0}$ being an $S$-wave contact coupling at lowest order and $\mu$ of order $m_{\pi}$ or $\sim 100 \mathrm{MeV}$. In a generic EFT, $\mu \ll \Lambda$. Thus, the only difference is with $J_{0}$. This is a "natural" scenario or choice as $J_{0}$ is actually a fundamental and physical constant in the nonperturbative regime, no longer an ordinary renormalization scale. We will discuss other schemes in future works $[9]$.

With the foregoing preparations, we could examine some important theoretical issues in $N N$ low energy scattering. First, let us consider some theoretical predictions. To this end, we calculate effective range expansions (ERE) of $1 / T$ in various channels. We should remind that the following discussions are valid for contact potentials only, not directly applicable to the cases containing long range potentials such as the pion-exchange potentials for $N N$ systems. Later we will consider some speculations about such cases.

Let us start with the uncoupled cases, where the general form of ERE of $1 / T$ reads:

$$
\left.\operatorname{Re}\left\{-\frac{4 \pi}{M} \frac{p^{2 L}}{T_{L}}\right\}\right|_{p \rightarrow 0}=\left.p^{2 L+1} \cot \left(\delta_{L}(p)\right)\right|_{p \rightarrow 0}=-\frac{1}{a}+\frac{1}{2} r_{e} p^{2}+\sum_{k=2}^{\infty} v_{k} p^{2 k},
$$

with $a, r_{e}$ and $\left[v_{k}, k \geq 2\right]$ being functions of the couplings in corresponding channels and $\left[J_{0}, J_{2 m+1}, m>\right.$ $0]$. However, unlike the rest of $[J \ldots], J_{0}$ contributes in each channel to only one of the ERE parameters $\left\{a, r_{e}, v_{k}, k \geq 2\right\}$ that is the coefficient of $p^{2 L}$ ! This is obviously due to the special status of the fundamental parameter $\mathcal{I}_{0}$. Employing the scenario (8) we could qualitatively deduce that, all but one ERE parameters are naturally sized! The exceptional one might be unnaturally sized just because of the contribution of $J_{0}$. 
TABLE I: Naturalness/unnaturalness of ERE parameters in uncoupled channels

\begin{tabular}{c|c|c}
\hline \hline Channels & natural & (might be) unnatural \\
\hline${ }^{1} S_{0}$ & $\left\{r_{e}, v_{k}, k \geq 2\right\}$ & $a$ \\
\hline${ }^{1} P_{1}{ }^{3} P_{0},{ }^{3} P_{1}$ & $\left\{a, v_{k}, k \geq 2\right\}$ & $r_{e}$ \\
\hline${ }^{1} D_{2},{ }^{3} D_{2}$ & $\left\{a, r_{e}, v_{k}, k \geq 3\right\}$ & $v_{2}$ \\
\hline$\cdots(L \geq 3)$ & $\left\{a, r_{e}, v_{k}, k \geq 2, k \neq L\right\}$ & $v_{L}$ \\
\hline \hline
\end{tabular}

The mechanism is simply that

$$
J_{0}+\left.\frac{d^{L}\left(\frac{N_{L}}{D_{L}}\right)}{L !\left(d p^{2}\right)^{L}}\right|_{p=0} \sim \frac{M}{4 \pi} \mathcal{O}(\mu)
$$

provided that the sign of $\left.\frac{d^{L}\left(N_{L} / D_{L}\right)}{L !\left(d p^{2}\right)^{L}}\right|_{p=0}$ is opposite to that of $J_{0}$ as closer analysis shows that $\left.\frac{d^{L}\left(N_{L} / D_{L}\right)}{L !\left(d p^{2}\right)^{L}}\right|_{p=0}$ is of the same magnitude as $J_{0}$. These general conclusions are summarized in Table I. It is known that in ${ }^{1} S_{0}$ channel the scattering length is unnaturally large with the rest of ERE parameters being natural. Now, within the context of contact potentials, higher ERE parameters might also be unnaturally sized in an appropriate channel. These are new predictions.

While in the coupled channels, one could find from Eq.(6) that the diagonal entries of $\mathbf{T}$ take the following form:

$$
\frac{1}{T_{L}}=\mathcal{I}_{0}+\frac{\mathcal{N}_{L ; 0}+\mathcal{I}_{0} \mathcal{N}_{L ; 1}}{\mathcal{D}_{L ; 0}+\mathcal{I}_{0} \mathcal{D}_{L ; 1}} p^{-2 L}
$$

where $J_{0}$ enters into the rational terms, and hence precluding a clear naturalness picture of the ERE parameters. However, using the scenario of $(8)$ and the detailed contents of $[\mathcal{N} \ldots, \mathcal{D} \ldots]$, one could still arrive at modestly good judgements, the status of naturalness in the coupled channels is basically similar to that given in Table I. There might be some deviations as $J_{0}$ now enters the rational terms, which would affect the status of some ERE parameters. But such influence would not be universal for all the ERE parameters. For example, through concrete calculations, one could find that in ${ }^{3} S_{1}$ channel, $r_{e}$ is totally independent of $J_{0}, a$ is most strongly influenced by $J_{0}$, while the rest of ERE parameters are only weakly affected due to the suppression just mentioned. More detailed analysis will be given in a forthcoming report [9].

In earlier EFT treatments, the distinctive aspects of nonperturbative renormalization demonstrated above were not fully appreciated, leading to quite some debates[1] (for recent debates, see [2, 3, 4]). A number of different schemes were proposed in order to remove the 'inconsistency' of Weinberg's power counting 2, 11, 19], with some "perturbative-like" expansion schemes being advanced [11, 19]. As is pointed out above, the inconsistency is in fact a misinterpretation of the failure of conventional wisdom of renormalization. Specifically, nonperturbative counterterms do not need to follow EFT power counting. Therefore, it is both difficult and unnecessary to maneuver a unified power counting [2, 4] . The entanglement property means that the problems could well be resolved with appropriate choice of nonperturbative prescriptions constrained by physical boundaries or conditions. After all, the ultimate goal of any sensible scheme or prescription should be to approach the physical dependence of $T$-matrices upon $p$ as far as possible. Thus, a (new) formally consistent power counting is not the full story: The nonperturbative prescription must be appropriately defined to match physical boundaries. For example, for a "perturbative-like" expansion scheme to work, the following two criteria must be satisfied: (1) The expansion converges; (2) Physical boundaries are fulfilled. Both criteria are dependent upon prescription choice. To illustrate this, we expand $1 / T_{L}$ in Eq. (5) as follows:

$$
\frac{1}{T_{L}}=\mathcal{I}_{0}+\frac{1+\delta N_{L}}{D_{L}^{0}+\delta D_{L}} p^{-2 L} \simeq \frac{1}{T_{L}^{0}}+\mathcal{O}\left(\frac{\delta N_{L}}{D_{L}^{0}}, \frac{\delta D_{L}}{D_{L}^{0}}\right) p^{-2 L}
$$

with $T_{L}^{0} \equiv\left(\mathcal{I}_{0}+\left(1 / D_{L}^{0}+\Delta_{L}\right) p^{-2 L}\right)^{-1}$ being the starting nonperturbative amplitude. Then, convergence requires that $\left|T_{L}^{0} \mathcal{O}\left(\delta N_{L} / D_{L}^{0}, \delta D_{L} / D_{L}^{0}\right) p^{-2 L}\right| \ll 1$, which in turn demands a sophisticated renormalization 
prescription after the couplings are given. Next, prescription must also be so chosen that $T_{L}$ fulfills physical boundaries. Therefore, it is a challenging task to find a prescription to fulfill the above two criteria.

Evidently, the informative form of the $T$-matrices will inspire new investigations in the future. It would be interesting to explore the relations between the nonperturbative parametrization elaborated here and those in literature, for example, the subtractive approaches [12, 13, 20, 21], and the lattice approaches [22].

Now we conclude with the following remarks. In general, the ultimate goal of a field theoretical calculation in nonperturbative regime is to identify and parametrize all the elements that govern the physical behaviors of the corresponding objects, especially the elements hidden in divergences. To this end, we have achieved the following: First, a fundamental parameter masked by a divergent integral was identified and shown to be RG invariant and inherent in all channels; Second, universal forms of nonperturbative $T$-matrices with respect to prescription dependence were obtained in all channels in the case of contact potentials; Third, within the realm of contact potentials, a simple scenario led us to predict that all the scattering lengths except those in the $S$-channels' are natural, while higher ERE parameters like $r_{e}, v_{k}, k \geq 2$ might also be unnatural in appropriate higher channels; Fourth, some distinctive notions about nonperturbative renormalization were revealed along with the failures of the conventional wisdoms, providing a different resolution of the intriguing problem with Weinberg's power counting in the EFT approach of $N N$ scattering. These conceptual gainings are significant from purely theoretical standpoint as nonperturbative renormalization is a challenging issue. Finally, we stress again that the notions and conclusions presented here are fairly general and hence illuminating for nonperturbative treatments of any systems dominated by short-distance interactions.

\section{Acknowledgement}

The author is deeply grateful to the anonymous referees for their valuable comments that significantly improved the presentation of our manuscript. The project is supported in part by the National Natural Science Foundation of China under Grant Nos. 10205004 and 10475028 and the Ministry of Education of China.

[1] P. Bedaque, U. van Kolck, Ann. Rev. Nucl. Part. Sci. 52, 339 (2002); E. Epelbaum, Prog. Part. Nucl. Phys. 57, $654(2006)$.

[2] A. Nogga, R.G.E. Timmermans, U. van Kolck, Phys. Rev. C72, 054006 (2005).

[3] M. Pavon Valderrama, E. Ruiz Arriola, Phys. Rev. C74, 064004 (2006); M.C. Birse, ibid.,C74, 014003 (2006).

[4] E. Epelbaum, Ulf-G. Meissner, arXiv: nucl-th/0609037.

[5] S. Weinberg, Phys. Lett. B 251, 288 (1990); Nucl. Phys. B 363, 1 (1991).

[6] J.-F. Yang, J.-H. Huang, Phys. Rev. C71, 034001, 069901(E) (2005).

[7] E. Braaten, H.W. Hammer, Phys. Rep. 428, 259 (2006).

[8] D.R. Phillips, S.R. Beane, T.D. Cohen, Ann. Phys. (NY) 263, 255 (1998).

[9] J.-F. Yang, forthcoming.

[10] M.C. Birse, J.A. McGovern, K.G. Richardson, Phys. Lett. B464, 169 (1999).

[11] D.B. Kaplan, M.J. Savage, M.B Wise, Phys. Lett. B424, 390 (1998); Nucl. Phys. B534, 329 (1998).

[12] C.-J. Yang, C. Elster, D.R. Phillips, Phys. Rev. C77, 014002 (2008).

[13] D.R. Entem, E. Ruiz Arriola, M. Pavon Valderrama, R. Machleidt, Phys. Rev. C77, 044006 (2008).

[14] G.P. Lepage, arXiv: nucl-th/9706029.

[15] T.-S. Park, K. Kubodera, D.-P. Min, M. Rho, Phys. Rev. C58, R637 (1998).

[16] E. Epelbaum, W. Glöckle, U. Meissner, Nucl. Phys. A671, 295 (2000); Eur. Phys. J. A19, 125,401 (2004).

[17] D.R. Entem, R. Machleidt, Phys. Rev. C66, 014002 (2002), ibid., C68, 041001 (2003).

[18] S.K. Bogner, et al, Phys. Lett. B576, 265 (2003); S.K. Bogner, R.J. Furnstahl, Phys. Lett. B632, 501 (2006).

[19] S.R. Beane, P. Bedaque, M.J. Savage, U. van Kolck, Nucl. Phys. A700, 377 (2002).

[20] T. Frederico, V.S. Timóteo, L. Tomio, Nucl. Phys. A653, 209 (1999).

[21] D. Eiras, J. Soto, Eur. Phys. J. A17, 89 (2003).

[22] S.R. Beane, P.F. Bedaque, K. Orginos, M.J. Savage, Phys. Rev. Lett. 97, 012001 (2006). 